\title{
Expression of the insect metalloproteinase inhibitor IMPI in the fat body of Galleria mellonella exposed to infection with Beauveria bassiana
}

\author{
Lidiia Vertyporokh and Iwona Wojda \\ Maria Curie-Sklodowska University, Faculty of Biology and Biotechnology, Institute of Biology and Biochemistry, Department of Immunobiology, \\ Lublin, Poland
}

The inducible metalloproteinase inhibitor (IMPI) discovered in Galleria mellonella is currently the only specific inhibitor of metalloproteinases found in animals. Its role is to inhibit the activity of metalloproteinases secreted by pathogenic organisms as virulence factors to degrade immune-relevant polypeptides of the infected host. This is a good example of an evolutionary arms race between the insect hosts and their natural pathogens. In this report, we analyze the expression of a gene encoding an inducible metalloproteinase inhibitor (IMPI) in fat bodies of the greater wax moth larvae Galleria mellonella infected with an entomopathogenic fungus Beauveria bassiana. We have used a natural infection, i.e. covering larval integument with fungal aerospores, as well as injection of fungal blastospores directly into the larval hemocel. We compare the expression of IMPI with the expression of genes encoding proteins with fungicidal activity, gallerimycin and galiomycin, whose expression reflects the stimulation of Galleria mellonella defense mechanisms. Also, gene expression is analyzed in the light of survival of animals after spore injection.

Key words: Insect immunity, Galleria mellonella, Beauveria bassiana, IMPI

Received: 17 June, 2016; revised: 12 October, 2016; accepted: 14 October, 2016; available on-line: 11 April, 2017

e-mail: wojda@hektor.umcs.lublin.pl

Abbreviations: AMPs, antimicrobial peptides; IMPI, inducible metalloproteinase inhibitor; PAMPs, pathogen associated molecular patterns; TIMP, tissue inhibitors of metalloproteinases

\section{INTRODUCTION}

The ability to clear infection is a precondition for survival of all organisms. Insects living in different habitats are exposed to many pathogens, including bacteria, fungi, and viruses, and despite possessing only innate immune mechanisms they are well protected against intruders. After recognition of pathogen associated molecular patterns (PAMPs), they switch on cellular and humoral immune response (Cytryńska et al., 2016). The former one comprises phagocytosis performed by single hemolymph cells called hemocytes and by trapping intruders in multihemocyte structures called capsules and nodules. Additionally, as a second line of defense, insects activate pathways regulating expression of defense peptides, called antimicrobial peptides (AMPs). These effector molecules act mostly by destabilization and perforation of microbial cellular membrane (Shai et al., 1999). They can be synthesized by different tissues but AMPs secreted to the hemolymph as part of the systemic im-

mune response are mostly produced by the insect fat body. On the other hand, entomopathogens, including fungi, have developed mechanisms that allow them to force the insect anatomical barriers and, while inside the host - destroy its defense molecules (Barelli et al., 2015; Valero-Jimenez et al., 2016). Among such virulence factors, there are proteases secreted inside the body of the infected insect, where they digest immune-relevant proteins and peptides (Travis et al., 1995; Wojda \& Taszłow, 2013). As both, hosts and pathogens, constantly undergo antagonist co-evolution, insects are forced to modulate their protective mechanisms. One of the most important findings of recent years was the discovery of an inducible metalloproteinase inhibitor (IMPI) in the insect model organism - the greater wax moth Galleria mellonella (Clermont et al., 2004). This is the first and so far the only specific inhibitor of microbial metalloproteinases. It shares no similarity with the tissue inhibitors of metalloproteinases (TIMPs) responsible for matrix degradation during development and morphogenesis, or with any known invertebrate or vertebrate proteins. The IMPI gene encodes a larger protein containing a furin cleavage site (Wedde et al., 2007). The IMPI protein product is a glycosylated, heat-stable, $8.6 \mathrm{kDa}$ molecule containing five intermolecular disulfide bonds. This protein inhibits the activity of microbial metalloproteinases such as thermolysin, but in contrast to TIMPs, it does not inhibit matrix metalloproteinases (MMPs, Wedde et al., 1998; 2007). This suggests that its main function is protection of $G$. mellonella against pathogen-secreted virulence factors. It has been shown that metalloproteinases, while secreted into the hemolymph of infected larvae, digest many hemolymph proteins into peptides, called protfrags, which, in turn, together with PAMPs, co-stimulate the insect immune system, acting probably as a danger signal (Griesh et al., 2000).

Beauveria bassiana is one of the fungal pathogens that have evolved mechanisms to infect and develop in many arthropods including insects. It is able to force the insect integument and get access to the insect body. After attaching to the cuticle, the fungal propagule germinates, creates an appressorium, and develops a penetration peg, producing enzymes like proteases, chitinases, and lipases, which digest the cuticle. Interestingly, when insects are regularly exposed to infection with this fungus, they modulate their integumental front line defense strategy and become more resistant to the entry of the pathogen into the body (Dubovskiy et al., 2013). Inside the hemocel, the fungus grows as blastospores with a thinner cell wall than that of cells growing in vitro. This is targeted at minimizing the recognition of infection by the PRR (pathogen recognition receptors) of the host. 
While infection progresses, the intruder secretes many virulence factors such as beauvericin, bassianolide, and oosporein (Ortiz-Urquiza et al., 2010; 2016; Butt et al., 2016). The infected animals die of the so-called white muscardine disease within a few days. Galleria mellonella larvae can also be infected by fungal propagules via injured cuticle, which is an infection gate leading directly to the larval hemocel. Spores of Beauveria bassiana have been used for many years for the production of biopesticides to protect crops against pests (Ortiz-Urquiza et al., 2015).

Galleria mellonella is one of the generally accepted insect models to study the insect immune response. Although its entire genome is not known, there is an increasing number of immune-relevant proteins and peptides identified in this organism (for the review, see Wo$\mathrm{jda}, 2016)$. Due to the size of the larvae, it is possible to obtain the fat body for RNA isolation and estimation of the amount of transcripts in this particular organ. Determination of IMPI expression profiles would provide us with important information concerning insect immune strategies. In this report, we test whether the expression of IMPI is part of an inducible immune response of Galleria mellonella infected with entomopathogenic fungus Beauveria bassiana. We followed the expression of the IMPI gene in the larval fat body after natural infection and after injection of fungal blastospores directly into the larval hemocel. We compare this expression with the expression levels of antifungal peptides: gallerimycin and galiomycin, which are known to be induced and secreted into the hemolymph in response to infection (Fehlbaum et al., 1994; Schuhmann et al., 2003; Lee et al., 2004). Their increased expression reflects the activation of $G$. mellonella immune response.

\section{MATERIALS AND METHODS}

Insects and infection thereof with Beauveria bassiana. Galleria mellonella (Lepidoptera: Pyralidae) was reared on honeybee nest debris in darkness at $28^{\circ} \mathrm{C}$ (stock), or at $25^{\circ} \mathrm{C}$ when used for fungal infection experiments. Beauveria bassiana strain 80.2, a kind gift from Bruno Lemaitre (CNRS, SGM, Gif-sur-Yvette, France), was grown on Sabouraud Dextrose Agar (1\% polypeptone, 4\% glucose, $1.5 \%$ agar) supplemented with a $2 \%$ yeast extract. For natural infection, each larva of $200 \mathrm{mg}$ weight was gently rolled on the surface of the sporulating fungus (Lemaitre et al., 1997) and left on the plate with the fungus for 6 minutes. The number of aerospores attached to the cuticle was determined by washing them out from the cuticle of 10 larvae with the use of dichloromethane (Ment et al., 2010) and was estimated to be 2.6 $( \pm 0.6) \times 10^{6}$ per larvae.

For blastospore preparation, SL YPD medium (glucose $4 \%$, bactopepton $2 \%$, yeast extract $0.5 \%$ ) was inoculated with $B$. bassiana and incubated for 3 days at $25^{\circ} \mathrm{C}$ with gentle shaking. The culture was then filtered through a sterile miracloth, centrifuged at $8000 \times g$ for $10 \mathrm{~min}$, washed with sterile apyrogenic water, and kept in water at $-20^{\circ} \mathrm{C}$. The concentration of blastospores was estimated by counting in a Burker chamber and additionally by counting CFU - colony forming units, by plating fungal blastospores on Sabouroud plates. The blastospores were diluted to the desired concentration in apyrogenic water and $5 \mu$ l of this mixture was injected into the larval hemocel. Before injection, the site of the injury was sterilized with $70 \%$ ethanol.

RNA isolation, reverse transcription, and Real Time qPCR. Galleria mellonella larvae were quick- ly cooled down in ice-cold water and sterilized in $70 \%$ ethanol. The fat bodies were isolated under ice-cold Ringer's solution $(172 \mathrm{mM} \mathrm{KCl}, 68 \mathrm{mM} \mathrm{NaCl}, 5 \mathrm{mM}$ $\mathrm{NaHCO}_{3}$, pH 6.1, osmolarity $420 \mathrm{mOsm}$ ), washed with the same solution, and placed in liquid nitrogen in Eppendorf tubes. Isolated organs were kept at $-70^{\circ} \mathrm{C}$ for RNA extraction. For each time-point, the organs from 5 larvae collected in each experiment were used for RNA extraction. Quantitative Real Time PCR was performed as described previously, using the StepOnePlus PCR System (Applied Biosystems), and Power SYBR Green PCR Master Mix (Life Technology) under conditions described previously (Wojda \& Taszłow, 2013; Vertyporokh et al., 2015). The primers for housekeeping gene S7e and for IMPI, gallerimycin, and galiomycin were described previously (Wojda \& Jakubowicz, 2007; Wojda \& Taszłow, 2013). The amount of mRNA was normalized to ribosomal protein S7e mRNA (gene/S7e) and shown as a relative value with respect to the values obtained from naive larvae. All data were collected from at least three assays. Statistical analysis was performed by Student's t-test and significant differences between indicated samples were established at $p<0.05$.

Analysis of the survival rate of $G$. mellonella injected with $B$. bassiana blastospores. Time-dependent larval survival was estimated using the Kaplan-Meier technique and comparison between curves was done with the Log-rank test (Goel et al., 2010).

\section{RESULTS}

Galleria mellonella larvae were naturally infected by covering the surface of the larval bodies with $B$. bassiana spores as described in the Materials and Methods section, without any injury of the cuticle. We followed the expression of IMPI in the fat bodies every 24 hours. As can be seen in Fig. 1, 48 hours post-infection we observed an almost 2 -fold increase in the expression of IMPI in the animal fat bodies, in comparison to its level in naive larvae. This amount of transcripts decreased in a step-wise manner with the development of fungal infection. To make sure that this natural infection triggered the insect immune response, we measured the level of mRNA for antifungal peptides, gallerimycin and galiomycin, which are known to be induced after a fungal infection (Wojda et al., 2009). We detected a step-wise increase in the level of gallerimycin. Also, the expression of galiomycin was induced and detected at all time-points after natural infection. Induction of galiomycin was previously shown to be faster than that of gallerimycin after natural infection with B. bassiana (Wojda et al., 2009).

Further, Galleria mellonella was infected by injection of $10^{1}, 10^{2}$, and $10^{3} \mathrm{~B}$. bassiana blastospores per larvae. We measured the expression of the IMPI gene 24 hours post-injection. We noticed that the injection of $10^{2}$ and $10^{3}$ blastospores induced the expression of antifungal peptides, although at this time-point the maximum expression was already observed when $10^{2}$ spores entered the larval hemocel. In the case of IMPI, the highest expression level was observed after the injection of $10^{2}$ blastospores, but it was reduced when $10^{3}$ spores were injected (Fig. 2).

We also followed the time-course of gene expression using two doses of fungal spores. As can be seen (Fig. 3A), when $10^{1}$ spores were injected, an increase in the IMPI expression was observed only at the 24-hour time-point, however it merely reached the level observed after the injection of water (compare with Fig. 2), 

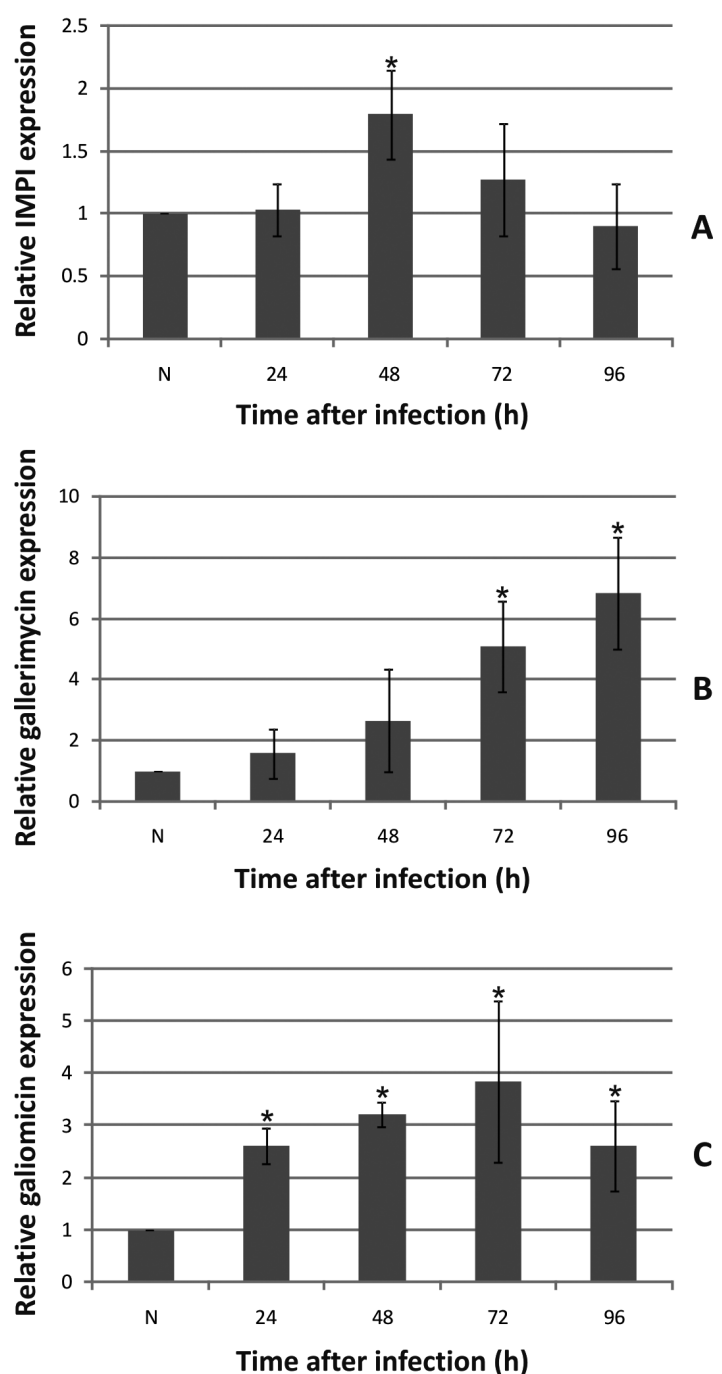

Figure 1. Relative RT-qPCR analysis of IMPI (A), gallerimycin and galiomycin ( $B$ and $C$, respectively) transcripts in the fat bodies of Galleria mellonella larvae naturally infected with the entomopathogenic fungus Beauveria bassiana.

The fat bodies were isolated from naive larvae $(\mathrm{N})$ and from larvae naturally infected with the fungus at the indicated time-points after infection. The data are shown as relative values with respect to naive larvae \pm S.D. and the asterisks $\left(^{*}\right)$ show significant differences.

while time-dependent induction of both gallerimycin and galiomycin was observed in the same animals, reaching the maximum 96 hours after injection. When the animals were infected with $10^{3}$ blastospores, expression of all tested genes was observed after 24 and 48 hours; then, it decreased after 72 hours (Fig. 3B).

We analyzed the results in a context of survival analysis of Galleria mellonella larvae injected with fungal spores. When $10^{1}$ blastospores were injected, 72 hours later, all animals were still alive and it was long before they began to die. Interestingly, even this small dose of injected spores caused the death of all larvae within 144 hours. When the 100-fold higher spore dose was used for infection, the animals began to die immediately after the 72-hour time-point. All of them died within 79 hours (Fig. 4).

\section{DISCUSSION}

The fungus Beauveria bassiana is a pathogen of the greater wax moth Galleria mellonella. As we had published
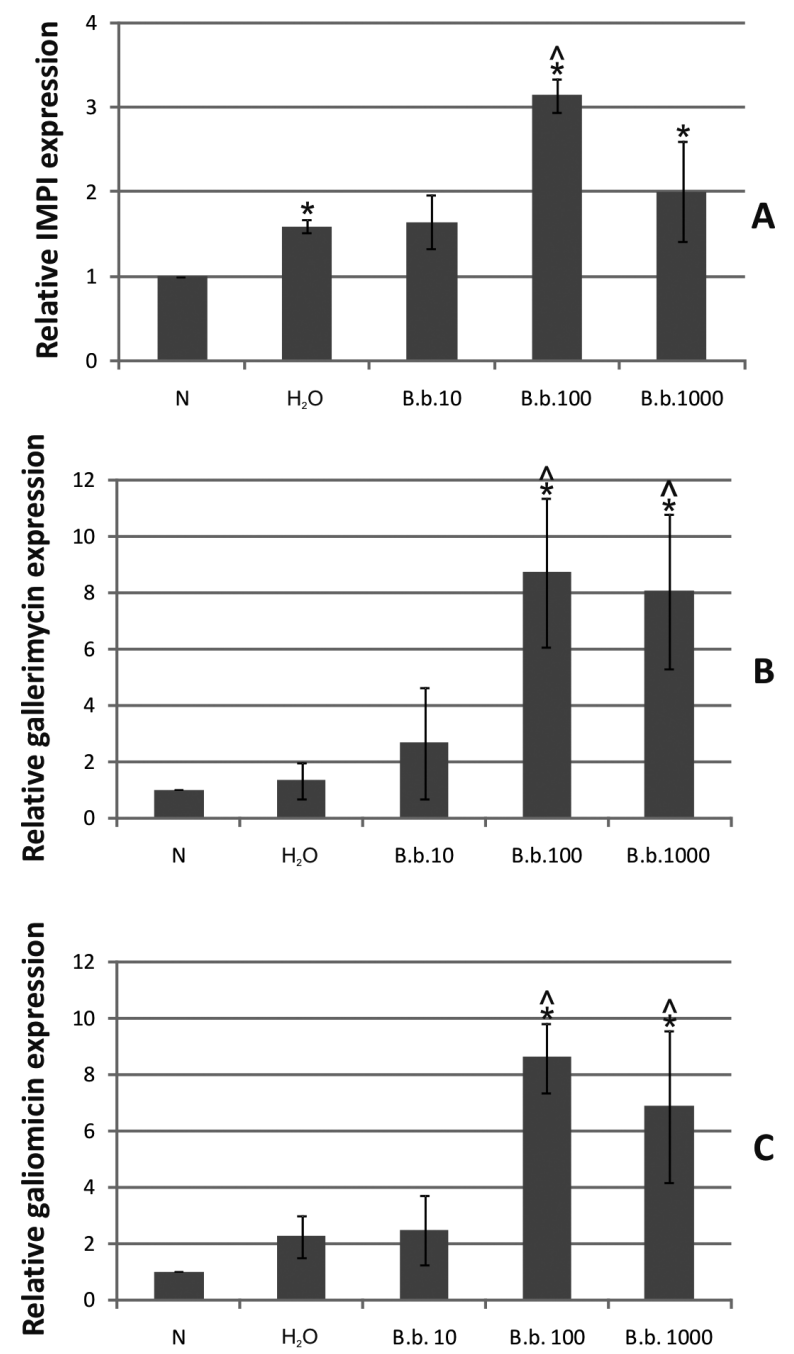

Figure 2. Expression of IMPI (A) and antifungal peptides (gallerimycin and galiomycin, $B$ and $C$, respectively) in the fat bodies of G. mellonella larvae injected with $10^{1}, 10^{2}$, and $10^{3}$ B. bassiana blastospores.

The fat bodies from naive larvae $(\mathrm{N})$ and larvae injected with apyrogenic water $\left(\mathrm{H}_{2} \mathrm{O}\right)$ or the indicated number of fungal (B.b.) spores, were collected 24 hours after injection. The data are shown as relative values with respect to naive larvae \pm S.D. Significant differences with respect to naive larvae $(p<0.05)$ or with respect to values from larvae injected with water are indicated by an asterisk $\left(^{*}\right)$ or by a dash $(\wedge)$, respectively.

previously, naturally infected larvae die within 216 hours of infection (Wojda et al., 2009). The results presented above show that IMPI expression is part of the humoral immune response of $G$. mellonella to infection with the mentioned fungus, although with different patterns of expression in comparison to the antifungal peptides: gallerimycin and galiomycin. Natural infection caused only weak induction of the metalloproteinase inhibitor, observed after 48 hours, while time-dependent induction of genes encoding defense molecules was observed. This suggests that the antifungal peptides and IMPI may be, at least in part, differently regulated. Induction of the immune response is triggered after detection of nonself like PAMPs (pathogen associated molecular patterns) and by DAMPs (damage associated molecular patterns), the latter also called the danger or damage signals (Krautz et al., 2014). In the latter case, this signal could come from the damaged cuticle, which occurs when spores of $B$. bassi- 


\section{B.bassiana 10 blastospores}
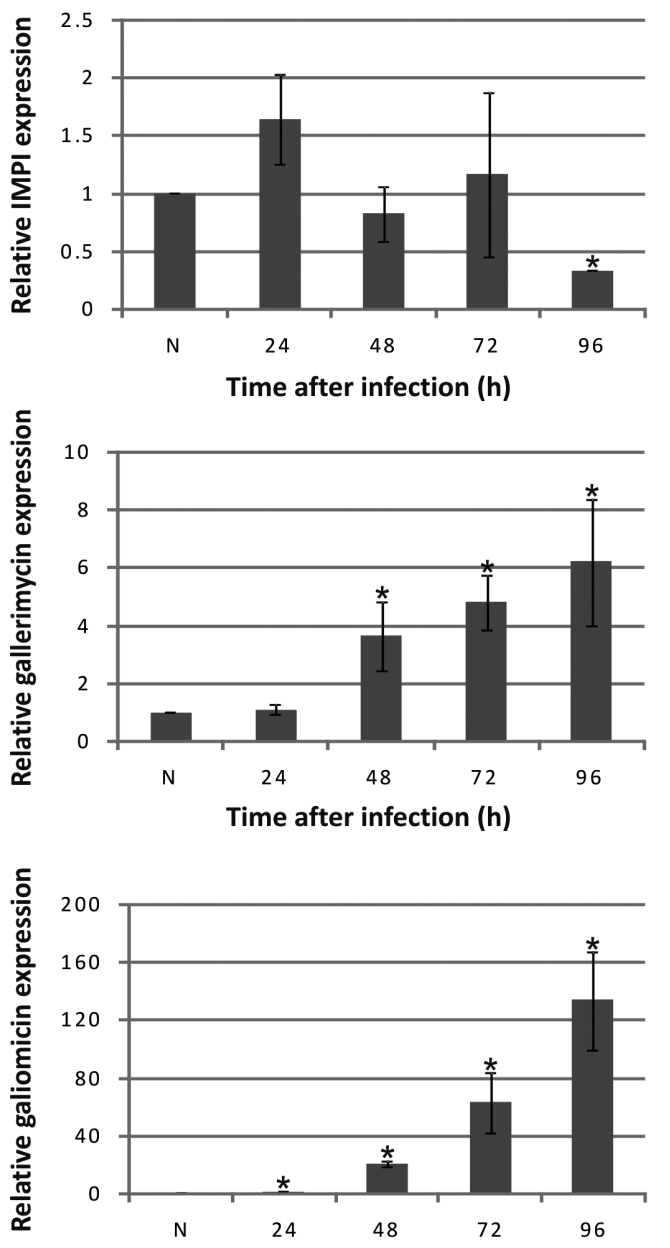

Time after infection (h)
B.bassiana 1000 blastospores
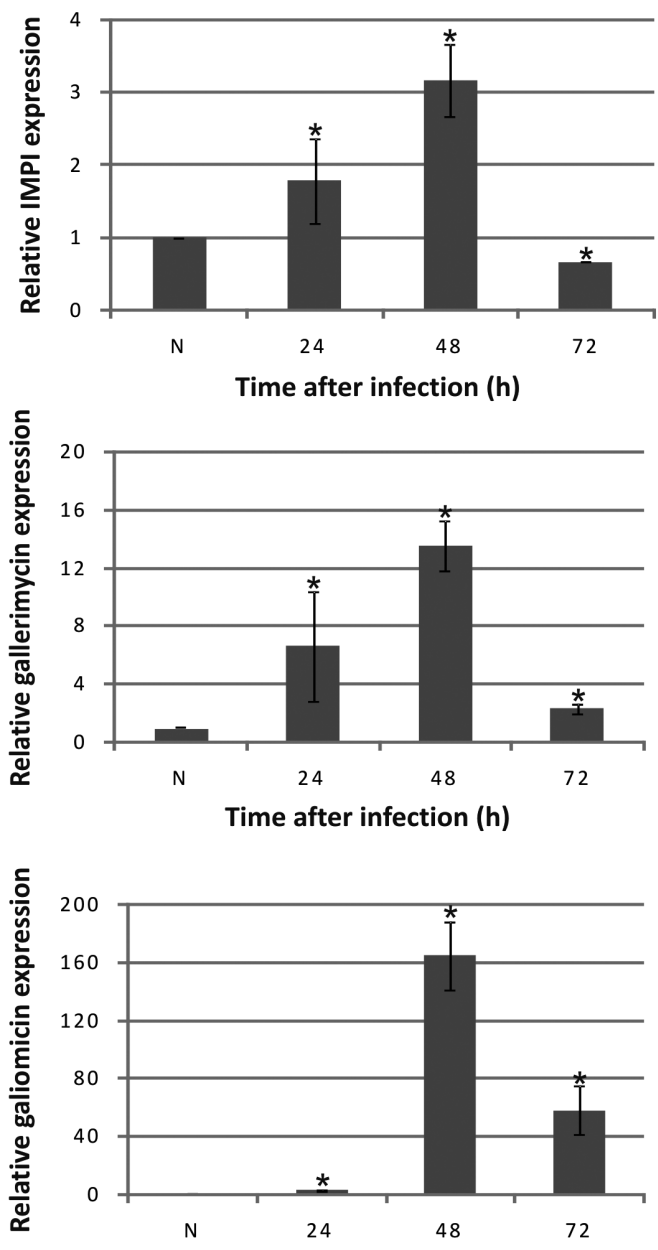

Time after infection (h)

Figure 3. The kinetics of the expression of IMPI (top) and genes encoding antifungal proteins, gallerimycin and galiomycin (middle and bottom, respectively), after injection of $10^{1}$ (A) and $10^{3}$ (B) B. bassiana spores.

The given values are relative with respect to values obtained for naive larvae ( \pm S.D.); significant differences are marked with an asterisk $(*)$.

ana force the insect integument and/or from destroyed internal structures of the infected host. Proteins, chitin, and lipids digested by proteases, chitinases, and lipases, respectively, may trigger the defense mechanisms of the infected host (Travis et al., 1995; Griesh et al., 2000). Among proteases secreted by B. bassiana, there is a subtilisin-like protease CDEP1, which has a big impact on the fungus virulence (Fan et al., 2010). Also, the B. bassiana genome encodes 98 metallopeptidases (Xiao et al., 2012). All of these factors may have a different influence on the expression of antifungal peptides and IMPI. In general, the induction of IMPI was more modest after natural infection, reaching only a 2 -fold increase, while after spore injection it increased by 3 fold at $48 \mathrm{~h}$ after the injection of $10^{3}$ bacterial spores. Here, it is worth mentioning that after injection of Candida albicans cells, which is an opportunistic pathogen for G. mellonella, induction of the IMPI gene was more than 5-fold in relation to its level in PBS injected larvae (Bergin et al., 2006). We have detected a nearly 8 -fold induction of the IMPI gene after $C$. albicans application and 16- fold induction after injection of Escherichia coli cells directly into the larval hemocel (Taszłow, Vertyporokh and Wojda, unpublished data). This means that the expression of
IMPI does not reach its maximum after infection with B. bassiana.

Expression of galiomycin, which was by 4 -folds greater after natural infection, reached a 130-160 fold increase while the infection was developing (96 and 48 hours after the injection of $10^{1}$ and $10^{3}$ spores, respectively). The injection of only $10^{1}$ fungal spores was not able to induce the expression of IMPI, gallerimycin, or galiomycin after 24 hours. Nevertheless, induction of both, gallerimycin and galiomycin, was observed while this infection proceeded. This was probably caused by multiplication of fungal spores in the larval hemocel. However, IMPI was not induced during the course of infection when the infection began with only $10^{1}$ fungal spores. This could be caused by dose-dependent differences in the kinetics of the host-pathogen interaction. It is possible that the number of fungal blastospores did not reach a threshold at the appropriate time to induce transcription of the IMPI gene. It is known that infected G. mellonella regulates the repertoire of defense molecules to maximize the total defense activity in the hemolymph, which is a result of synergistic action of hemolymph components (Bolouri-Moghaddam et al, 2016). However, it is worth mentioning that Galleria mellonella larvae injected with only 


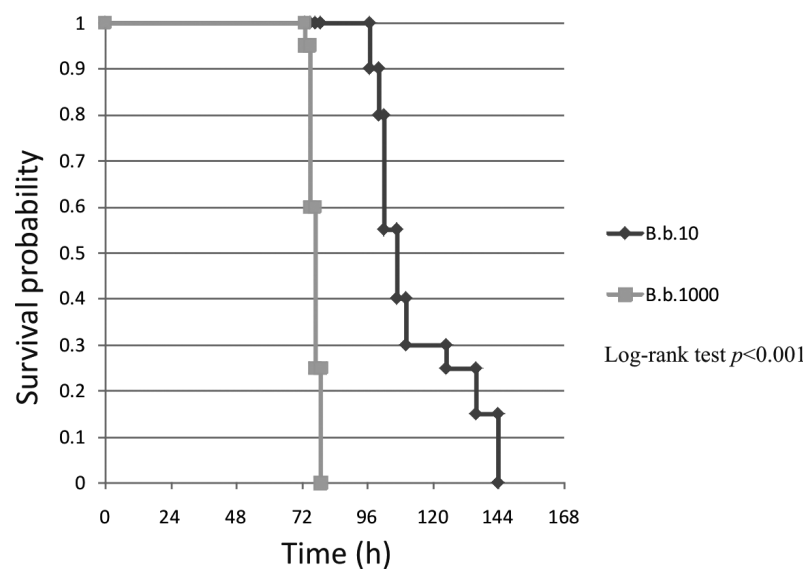

Figure 4. Kaplan-Meier analysis of Galleria mellonella larvae survival after injection of different doses of Beauveria bassiana spores.

Survival analysis was performed on the total number of 100 larvae in each group. Comparison between curves was done by the logrank test.

$10^{1}$ fungal spores finally died within 144 hours. The expression of IMPI was induced in time when $10^{3}$ spores were injected into the larval hemocel. In this case, induction of all of the 3 genes tested occurred at 24 and 48 hours, and dropped at the $72 \mathrm{~h}$ time-point. The observed drop could be explained with the fact that, after 3 days, larvae injected with this dose of fungal spores began to die. They may have been too weak for the energy consuming transcription or the expression may have been inhibited by factors secreted by the entomopathogenic fungus. The latter seems to be more probable, because the expression of IMPI was higher when a lethal dose of $C$. albicans was injected, in comparison to a non-lethal dose, at a time point when animals injected with the lethal dose were already very week as well (Vertyporokh and Wojda, unpublished data).

Interestingly, about 1.5 increase in IMPI transcripts was observed 24 hours after mechanical stress was applied on G. mellonella larvae. This increased expression, together with the increased expression of galiomycin, was supposed to make the larvae less susceptible for further infection with C. albicans (Mowlds et al., 2008).

Inducible metalloproteinase inhibitor is postulated to be an outcome of the antagonist co-evolution between G. mellonella and pathogenic fungi. This unique protein has evolved as a part of defense mechanism against metalloproteinases secreted by invading organisms (Joop \& Vilcinskas, 2016). Taking into consideration that the protein product of the IMPI gene contains a furin-cleavage site, generating an $8.6 \mathrm{kDa}$ IMPI after limited proteolysis (Wedde et al., 2007), it is likely that the amount of specific metalloproteinase inhibitor in larval hemolymph can be also regulated at a post-translational level. Thus, it would be interesting to compare the relative changes in the IMPI transcripts with its level in the hemolymph when its specific antibodies become available.

Further research is needed to determine which factors regulate the expression of IMPI in comparison to other defense proteins and peptides.

\section{REFERENCES}

Barelli L, Moonjely S, Behie SW, Bidochka MJ (2015) Fungi with multifunctional lifestyles: endophytic insect pathogenic fungi. Plant Mol Biol 90: 657-664. doi: 10.1007/s11103-015-0413-z
Bergin D, Murphy L, Keenan J, Clynes M, Kavanagh K (2006) Pre-exposure to yeast protects larvae of Galleria mellonella from subsequent lethal infection by Candida albicans and is mediated by the increased expression of antimicrobial peptides. Microb Infect 8: 2105-2112

Bolouri Moghaddam MR, Tonk M, Schreiber C, Salzig D, Czermak P, Vilcinskas A, Rahnamaeian M (2016) The potential of the Galleria mellonella innate immune system is maximized by the co-presentation of diverse antimicrobial peptides. Biol Chem 397: 939-945

Butt TM, Coates CJ, Dubovskiy IM, Ratcliffe NA (2016) Entomopathogenic fungi: new insights into host-pathogen interaction in Genetics and Molecular Biology of Entomopathogenic Fungi. First Edition. Lovett B, St. Leger R, eds, pp 307-364. Elsevier

Clermont A, Wedde M, Seitz V, Podsiadlowski L, Lenze D, Hummel M, Vilcinskas A (2004) Cloning and expression of an inhibitor of microbial metalloproteinases from insects contributing to innate immunity. Biochem J 382: 315-322

Cytryńska M, Wojda I, Jakubowicz T (2016) How insects combat infections in Lessons in Immunit. From sinole-cell organisms to mammals. Ballarin L, Cammarata M eds. pp 117-124. Academic Print, Elsevier

Dubovskiy IM, Whitten MM, Yaroslavtseva ON, Greig C, Kryukov VY, Grizanova EV, Mukherjee K, Vilcinskas A, Glupov VV, Butt TM (2013) Can insects develop resistance to insect pathogenic fungi? PLOS One 8: e 60248. doi:10.1371/annotation/3c61c1d6-7981$4 \mathrm{f} 3 \mathrm{e}-\mathrm{a} 690-1 \mathrm{ce} 7 \mathrm{a} 4 \mathrm{~d} 89285$

Goel MK, Khanna P, Kishore J (2010) Understanding survival analysis: Kaplan-Meier estimate. Int J Ayurveda Res 1: 274-278. doi: 10.4103/0974-7788.76794

Griesch J, Wedde M, Vilcinskas A (2000) Recognition and regulation of metalloproteinase activity in the haemolymph of Galleria mellonella: a new pathway mediating induction of humoral immune responses. Insect Biochem Mol Biol 30: 461-472

Fan Y, Pei X, Guo S, Zhang Y, Luo Z, Liao X, Pei Y (2010) Increased virulence using engineered protease-chitin binding domain hybrid expressed in the entomopathogenic fungus Beauveria bassiana. Microb Pathog 49: 376-80. doi: 10.1016/j.micpath.2010.06.013

Fehlbaum P, Bulet P, Michaut L, Lagueux M, Broekaert WF, Hetru C, Hoffmann JA (1994) Insect immunity. Septic injury of Drosophila induces the synthesis of a potent antifungal peptide with sequence homology to plant antifungal peptides. J Biol Chem 269: 3315933163

Joop G, Vilcinskas A (2016) Coevolution of parasitic fungi and insect hosts. Zoology 119: 350-358

Krautz R, Arefin B, Theopold U (2014) Damage signals in the insect immune response. Front Plant Sci 5: e342. doi: 10.3389/ fpls.2014.00342

Lee YS, Yun EK, Jang WS, Kim I, Lee JH, Park SY, Ryu KS, Seo SJ, Kim CH, Lee IH (2004) Purification, cDNA cloning and expression of an insect defensin from the great wax moth, Galleria mellonella. Insect Mol Biol 13: 65-72

Lemaitre B, Reichhart JM, Hoffmann J.A. (1997) Drosophila host defense: Differential induction of antimicrobial peptide genes after infection by various classes of microorganisms. Proc Nat Acad Sci USA 94: 14614-14619.

Ment D, Gindin G, Rot A, Soroker V, Glazer I, Barel S, Samish M (2010) Novel technique for quantifying adhesion of Metarbizium anisopliae conidia to the tick cuticle. Appl Environ Microbiol 76: 35213528. doi: 10.1128/AEM.02596-09

Mowlds P, Barron A, Kavanagh K (2008) Physical stress primes the immune response of Galleria mellonella larvae to infection by Candida albicans. Microbes Infect 10: 628-634

Ortiz-Urquiza A, Riveiro-Miranda L, Santiago-Álvarez C, QuesadaMoraga E (2010) Insect-toxic secreted proteins and virulence of the entomopathogenic fungus Beauveria bassiana. I Invert Pathol 105: 270-178. doi: 10.1016/j.jip.2010.07.003

Ortiz-Urquiza A, Luo Z, Keyhani NO (2015) Improving mycoinsecticides for insect biological control. Appl Microbiol Biotechnol 99: 10571068. doi: 10.1007/s00253-014-6270-x

Ortiz-Urquiza A, Keyhani NO (2016) Molecular genetics of Beanveria bassiana infection of insects. Adv Gen 94: 165-249. doi: 10.1016/ bs.adgen.2015.11.003

Schuhmann B, Seitz V, Vilcinskas A, Podsiadlowski L (2003) Cloning and expression of gallerimycin, an antifungal peptide expressed in immune response of greater wax moth larvae. Galleria mellonella. Arch Insect Biochem Physiol 53: 125-133

Shai Y (1999) Mechanism of the binding, insertion and destabilization of phospholipid bilayer membranes by alpha-helical antimicrobial and cell non-selective membrane-lytic peptides. Biochim Biophys Acta 1462: 55-70

Travis J, Potempa J, Maeda H (1995) Are bacteria proteinases pathogenic factors? Trends in Microbiol 3: 405-407

Valero-Jiménez CA, Wiegers H, Zwaan BJ, Koenraadt CJ, van Kan JA (2016) Genes involved in virulence of the entomopathogenic fungus Beauveria bassiana. J Invert Pathol 133: 41-49. doi: 10.1016/j. jip.2015.11.011

Vertyporokh L, Taszłow P, Samorek-Pieróg M, Wojda I (2015) Shortterm heat shock affects the course of immune response in Galleria 
mellonella naturally infected with the entomopathogenic fungus Beauveria bassiana. J Invert Pathol 130: 42-51. doi: 10.1016/j.jip.2015.07.001

Wedde M, Weise C, Kopacek P, Franke P, Vilcinskas A (1998) Purification and characterization of an inducible metalloproteinase inhibitor from the hemolymph of the grater wax moth larvae. Galleria mellonella. Eur J Biochem 255: 535-543

Wedde M, Weise C, Rolf N, Altincicek B, Vilcinskas A (2007) The insect metallopreoteinase inhibitor gene of the lepidopteran Galleria mellonella encodes two distinct inhibitors. Biol Chem 388: 119-127.

Wojda I, Jakubowicz T (2007) Humoral immune response upon mild heat-shock conditions in Galleria mellonella larvae. I Insect Physiol 53: $1134-1144$
Wojda I, Kowalski P, Jakubowicz T (2009) Humoral immune response of Galleria mellonella larvae after infection by Beauveria bassiana under optimal and heat-shock conditions. J Insect Physiol 55: 525-531

Wojda I, Taszłow P (2013) Heat shock affects host-pathogen interaction in Galleria mellonella infected with Bacillus thuringiensis. I Insect Physiol 59: 894-905. doi: 10.1016/j.jinsphys.2013.06.011

Wojda I (2016) Immunity of the greater wax moth Galleria mellonella. Insect Sci. doi: 10.1111/1744-7917.12325

Xiao G, Ying SH, Zheng P, Wang ZL, Zhang S, Xie XQ, Shang Y, St Leger RJ, Zhao GP, Wang C, Feng MG (2012) Genomic perspectives on the evolution of fungal entomopathogenicity in Beauveria bassiana. Sci Rep 2: e483. doi: 10.1038/srep00483 